\title{
Anterior cingulate cortex and insula response during indirect and direct processing of emotional faces in generalized social anxiety disorder
}

Heide Klumpp ${ }^{1 *}$, David Post ${ }^{1}$, Mike Angstadt ${ }^{2}$, Daniel A Fitzgerald ${ }^{1}$ and K Luan Phan ${ }^{1,2,3}$

\begin{abstract}
Background: Generalized social anxiety disorder (gSAD) is associated with a heightened neural sensitivity to signals that convey threat, as evidenced by exaggerated amygdala and/or insula activation when processing face stimuli that express negative emotions. Less clear in the brain pathophysiology of gSAD are cortical top down control mechanisms that moderate reactivity in these subcortical emotion processing regions. This study evaluated amygdala, insula, and anterior cingulate cortex (ACC) activity in GSAD with a novel "Emotional Faces Shifting Attention Task" (EFSAT), an adaptation of perceptual assessment tasks well-known to elicit amygdala response. In healthy volunteers, the task has been shown to engage the amygdala when attention is directed to emotional faces and the ACC when attention is directed to shapes, away from emotional faces.
\end{abstract}

Methods: During functional MRI, 29 participants with gSAD and 27 healthy controls viewed images comprising a trio of faces (angry, fear, or happy) alongside a trio of geometric shapes (circles, rectangles, or triangles) within the same field of view. Participants were instructed to match faces or match shapes, effectively directing attention towards or away from emotional information, respectively.

Results: Participants with gSAD exhibited greater insula, but not amygdala, activation compared to controls when attending to emotional faces. In contrast, when attention was directed away from faces, controls exhibited ACC recruitment, which was not evident in gSAD. Across participants, greater ACC activation was associated with less insula activation.

Conclusions: Evidence that individuals with GSAD exhibited exaggerated insula reactivity when attending to emotional faces in EFSAT is consistent with other studies suggesting that the neural basis of GSAD may involve insula hyper-reactivity. Furthermore, greater ACC response in controls than GSAD when sustained goal-directed attention is required to shift attention away from social signals, together with a negative relationship between ACC and bilateral insula activity, indicate the ACC may have served a regulatory role when the focus of attention was directed to shapes amidst emotional faces.

Keywords: Social anxiety, fMRI, Emotional faces, Threat processing, Brain imaging

\footnotetext{
* Correspondence: hklumpp@psych.uic.edu

${ }^{1}$ Mood and Anxiety Disorders Research Program, Department of Psychiatry (HK, DP, DAF, KLP), University of Illinois at Chicago, 1747 W. Roosevelt Rd, Chicago, IL 60608, USA

Full list of author information is available at the end of the article
} 


\section{Background}

Generalized social anxiety disorder (gSAD) is characterized by extreme fears of potential scrutiny encompassing most social situations $[1,2]$ and, therefore, can lead to impaired functioning in educational, occupational, and interpersonal domains $[1,3,4]$. Clinical manifestations of gSAD reflect a heightened threat processing system. These include excessive attention to negative social-signals (e.g., angry, fearful facial expressions [5]); fear-based physiological responses (autonomic changes) in anticipation of [6,7], or during [8], anxiety-evoking situations; and negative predictions about social events [9].

Thus far, models of the neural pathophysiology of gSAD have focused on an enhanced threat processing system that have fundamentally evolved from amygdala-centric 'functional' brain activation paradigms. In light of the central role amygdala plays in mediating fear and threat-related processing [7,10-12], its response to emotional information has been a predominant focus in affective neuroscience. As one example, when coupled with functional neuroimaging, perceptual matching tasks are designed to isolate the influence of emotional face content by contrasting a matching face condition with a sensorimotor control condition (i.e., matching shapes) and to robustly elicit amygdala response in healthy volunteers [13-16]. Building on this paradigm, studies of gSAD have shown amygdala reactivity to threat exceeds that of healthy individuals $[17,18]$ and the extent of this reactivity has been shown to reflect symptom severity [19].

There is increasing evidence that the neural substrates of gSAD extend beyond the amygdala. In addition, there is a growing realization that functional neuroimaging paradigms should move beyond perceptual assessment tasks that primarily probe subcortical reactivity in key emotion processing regions. First, accumulating data point to insula hyper-reactivity in regard to processing negative emotional information in gSAD [20-27], and evidence of a correlation between anterior insula (aINS) reactivity to threatrelevant cues and symptom severity [24] also supports the notion that exaggerated aINS reactivity underlies anxiety disorders $[28,29]$. It has been posited that negative beliefs are mediated by feeling states, a core function of the aINS $[30,31]$. That is, aberrant insula activiation in anxiety is thought to be driven by sensitivity to aversive interoceptive signals and/or inaccurate interpretations of ordinary changes in bodily state $[28,29]$. Second, the prefrontal cortex (PFC) has reciprocal connections with the amygdala [32-34] and aINS [30,35-39], yet less is known about prefrontal mechanisms in gSAD. The relative gap in knowledge may relate to the interest in aberrant amygdala activation in anxiety and use of perceptual matching tasks, which are not well-validated to probe PFC regions.

Several functional neuroimaging tasks exist that are known to robustly recruit PFC areas by engaging cognitive functions such as 'top down' attentional control-that is, the ability to effectively resolve the type of conflict that occurs when cognitive goals compete with salient distractors for limited processing resources [40-48]. For example, to reflect the competition for processing resources imposed by distractors, tasks such as the emotional counting Stroop [48-50], modified dot probe detection [51,52], and "faces/houses" [40,53] have in common the rapid and simultaneous presentation of non-emotional, task-relevant stimuli and salient distractors (e.g., $500 \mathrm{~ms}$ or less; [40,51-54]). Studies using such tasks have shown that anxiety-prone $[40,41,55,56]$ and clinically anxious patients $[49,50,57]$, including those with gSAD [49], exhibit deficiencies in the recruitment of the anterior cingulate cortex (ACC) and other emotion regulation areas (e.g., dorsolateral PFC) in the presence of threat distractors. Excessive attention to threat appears to encompass impoverished top-down control at least when the window of information processing is markedly restricted (i.e., when visual processing is fast).

Current models propose that there is a balance between attending to the task at hand and to the emotional salience that surround the given task. Resolving emotional conflict must occur in the context that salient emotional cues not only capture but sustain visual attention [58]. Consequently, prefrontal areas should engage during the maintenance of goal-directed attention even when the window of information processing is extended to that of direct emotion processing.

In order to capture this balance, we adapted the face matching task into a relatively slow, easy-to-perform blocked perceptual matching task known as the "Emotional Faces Shifting Attention Task" (EFSAT), which requires subjects to shift their attention towards and away from emotional faces. In contrast to the traditional faces-only and shapes-only images, in EFSAT both image types were configured to be in the same field of view (Figure 1). Therefore, the instruction to "Match Shapes"

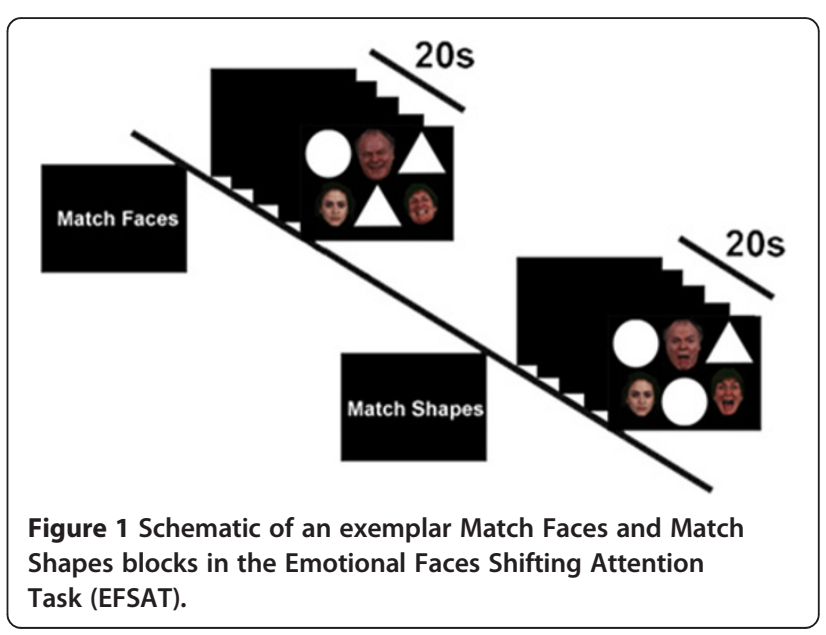


directed attention away from emotional faces whereas "Match Faces" directed attention towards emotional faces. Analogous to traditional perceptual assessment tasks, each matching trial was presented for $4 \mathrm{sec}$ in back-to-back blocks. In a recent study [59], we demonstrated the modification was successful as healthy volunteers engaged the amygdala to "Match Faces" and the rostral ACC to "Match Shapes" indicating the ACC effectively impeded the processing of task-irrelevant emotional faces presented alongside shapes. Thus, EFSAT complements traditional attentional control paradigms shown to recruit PFC areas.

In an effort to assess neural correlates of sustained attentional control in gSAD, participants performed the EFSAT during functional MRI. Our predictions for emotion processing (Match Faces $>$ Match Shapes) were individuals with gSAD compared to demographically matched healthy controls (HC) would demonstrate: 1) exaggerated amygdala and anterior insula reactivity, 2) a positive relationship between activation in these regions and symptom severity, and 3) subcortical effects would be most pronounced when the faces constituted social signals of threat (angry and fear). Regarding attentional control (Match Shapes $>$ Match Faces), we predicted gSAD compared to $\mathrm{HC}$ would exhibit: 1) reduced ACC activation, and 2) deficient ACC response would be most pronounced in the presence of distracting threat faces. Lastly, given reciprocal subcortical-ACC associations, we hypothesized insula activation to emotion processing would negatively correlate with ACC response during attentional control across participants.

\section{Methods}

\section{Participants}

Twenty-seven matched $\mathrm{HC}$ were recruited through community advertisements. The current $\mathrm{HC}$ group incorporates six new participants to the cohort in the previous
EFSAT study involving only healthy volunteers [59], which previously served as a validation of this novel task and prompted pursuing the current study and informed the stated hypotheses. Twenty-nine individuals diagnosed with gSAD were identified through community advertisement and an outpatient psychiatric clinic. Participants completed the Structured Clinical Interview for DSM-IV [60] conducted by a licensed psychologist in addition to measures of symptoms and negative mood, such as the Liebowitz Social Anxiety Scale "LSAS" [61] and Beck Depression Inventory "BDI" [62]. All of the subjects were free of psychotropic medications except for two individuals with gSAD who were taking a selective serotonin reuptake inhibitor. None of the participants with gSAD had a current major depressive episode or severe depression symptoms (i.e., BDI score of 30 or greater; [62]). Furthermore, none of the participants with gSAD had recent substance abuse/dependence (within 6 months of study) or a lifetime history of major psychiatric illness (e.g., bipolar, psychotic disorder). All participants were between 18 and 55 years of age, right-handed, and free of current and past major medical or neurologic illness, as confirmed by a Board Certified physician. None of the participants tested positive for alcohol or illegal substances. GSAD and HC groups were matched for age $(t(54)=0.02, p=0.99)$, gender $\left(\chi^{2}(1\right.$, $\mathrm{N}=56)=0.25, p=0.62)$, and race/ethnicity $\left(\chi^{2}(3, \mathrm{~N}=56)=\right.$ 4.54, $p=0.21$ ). All participants provided written informed consent, as approved by the Institutional Review Boards (ethics committee) of the University of Michigan Medical School in compliance with the Helsinki Declaration. See Table 1 for group characteristics.

\section{Emotional faces shifting attention task}

During fMRI scanning, participants performed the EFSAT, which consisted of trials depicting a trio of geometric shapes (circles, rectangles, triangles) presented

Table 1 Group characteristics (Mean \pm SD)

\begin{tabular}{|c|c|c|c|c|}
\hline & gSAD $(n=29)$ & $H C(n=27)$ & $t(d f=56)$ & $p$ \\
\hline Age (years) & $24.9 \pm 6.3$ & $24.9 \pm 5.9$ & 0.02 & 0.99 \\
\hline Gender & $18 \mathrm{~F} / 11 \mathrm{M}$ & $15 \mathrm{~F} / 12 \mathrm{M}$ & & \\
\hline Race/Ethnicity & $6 \mathrm{Asa} / 3 \mathrm{AA} / 2 \mathrm{H} / 18 \mathrm{C}$ & 3Asa/1AA/OH/23C & & \\
\hline Social Anxiety Severity & $77.3 \pm 15.4$ & $7.8 \pm 6.3$ & 21.69 & $<0.001$ \\
\hline State Anxiety Level & $42.9 \pm 8.6$ & $24.0 \pm 5.4$ & 9.72 & $<0.001$ \\
\hline Trait Anxiety Level & $49.1 \pm 8.4$ & $26.6 \pm 4.0$ & 12.69 & $<0.001$ \\
\hline Depression Level & $12.0 \pm 7.9$ & $0.67 \pm 1.0$ & 7.41 & $<0.001$ \\
\hline
\end{tabular}

gSAD, Generalized Social Anxiety Disorder participants.

$\mathrm{HC}$, Healthy Control participants.

$\mathrm{F}$, Female.

M, Male.

Asa, Asian American; AA, African American; H, Hispanic; C, Caucasian.

Social anxiety severity measured with the Liebowitz Social Anxiety Scale.

State and trait anxiety levels measured with the Spielberger State-Trait Anxiety Inventory.

Depression level measured with the Beck Depression Inventory. 
alongside a trio of faces within the same field of view (Figure 1). During the "Match Faces" condition, participants had to select from one of two bottom faces (one emotional vs. one neutral) which matched the emotional expression of the top target face, whereas in the "Match Shapes" condition, participants selected from the two top shapes the one matching the bottom target shape. The faces were chosen from a validated stimulus set [63], were presented without repetition, and equally represented both genders.

The task comprised 36 back-to-back blocks: 18 blocks of matching shapes interleaved with 18 blocks of matching emotional faces, counterbalanced across two runs. Each target emotional face condition (angry, fear, happy) was presented in six 20-second blocks; these were presented pseudo-randomly, without subsequent repetition of individual faces. Each block began with a 4 second cue to either "Match Faces" (attend to faces) or "Match Shapes" (attend away from faces) followed by the four sequential matching trials, each lasting 4 seconds. Participant responses were recorded via button press.

\section{Functional imaging: acquisition and analysis}

Functional imaging was performed with blood-oxygenlevel-dependent (BOLD) sensitive whole-brain fMRI on a 3.0 Tesla GE Signa System (General Electric; Milwaukee, WI) using a standard radio frequency coil. Images were acquired with 30 axial, 5 -mm-thick slices using a standard $\mathrm{T} 2$ *-sensitive gradient echo reverse spiral acquisition sequence $(2 \mathrm{~s}$ repetition time; $25 \mathrm{~ms}$ echo time; $64 \times 64$ matrix; $24 \mathrm{~cm}$ field of view; flip angle $77^{\circ} ; 3.75 \times 3.75 \times$ $5 \mathrm{~mm}$ final voxel size). A high-resolution, T1-weighted volumetric anatomical scan was also acquired for anatomical localization. Data from all participants met the criteria for quality with minimal motion correction (movements were less than $2 \mathrm{~mm}$ in any one direction across each functional run) and the first 4 volumes from each run were discarded to allow for T1 equilibration effects. Conventional preprocessing steps were used in Statistical Parametric Mapping (SPM8) software package (Wellcome Trust Centre for Neuroimaging, London www.fil.ion.ucl. ac.uk/spm). Briefly, images were temporally corrected to account for differences in slice time collection, spatially realigned to the first image of the first run, normalized to a Montreal Neurological Institute (MNI) template, and smoothed with an $8 \mathrm{~mm}$ isotropic Gaussian kernel.

A general linear model was applied to the time series, convolved with the canonical hemodynamic response function and with a $128 \mathrm{~s}$ high-pass filter. Blocks of Match Faces (shapes in 'background') and Match Shapes (faces in 'background') were modeled separately based on the target emotion or shape (angry, fearful, or happy / circle, square, or triangle) resulting in six regressors, the effects of which were estimated for each voxel for each participant and taken to the second level for random effects analysis.

A region of interest (ROI)-based Analysis of Variance (ANOVA) was conducted to test our a priori hypotheses-specifically, within the bilateral amygdala, anterior insula, and anterior cingulate cortex (ACC) as defined by atlas-based anatomical ROIs with Automated Anatomical Labeling (AAL) toolbox within SPM, in the context of main effects of Group (gSAD, HC), Emotion (angry, fear, happy), and Group by Emotion interaction. Activations were deemed significant at a p-value of $<0.05$, corrected for multiple comparisons within the $a$ priori anatomically-confined ROI (i.e., a small volume correction $[\mathrm{SVC}]$ ). For completeness, we also report activation clusters outside a priori ROIs at a whole-brain threshold of $\mathrm{p}<0.001$ uncorrected with at least 10 contiguous voxels.

To clarify the direction of activation and to examine correlations, parameter estimates of peak activation ( $\beta$ weights, arbitrary units [a.u.]) were extracted from spherical (10-mm diameter) ROIs from each participant and submitted to post hoc t-tests in the Statistical Package for the Social Sciences (SPSS) (Chicago, IL version 18).

Pearson correlational analyses were conducted to evaluate the predicted positive relationship between amygdala and/or insula activation with symptom severity, and the association between subcortical (amygdala, aINS) activation and ACC response.

Behavioral data were submitted to a 2 (Group: gSAD, HC) x 3 (Emotion: fear, angry, happy) ANOVA with repeated measures for the Emotion factor for Match Faces and emotional 'distractor' for Match Shapes. All significant main effects and interactions were followed up with two-tailed t-tests, $\mathrm{p}<0.05$.

\section{Results}

\section{Behavioral data: match faces}

For accuracy, results revealed a main effect of Emotion $[F(2,108) 4.41, p<0.016]$ but no main effect of Group or Emotion $x$ Group interaction (all $p_{s}>0.05$ ). The main effect of Emotion showed participants were more accurate at matching fearful than angry faces $(\mathrm{p}<0.007)$; no differences were evident for fearful versus happy $(\mathrm{p}=0.13)$ or angry versus happy faces $(p=0.12)$. Similarly, mean reaction times (RT) for accurate trials showed a main effect of Emotion $[F(2,108) 36.0, p<0.001]$ but no main effect of Group or Emotion $x$ Group interaction (all $p_{s}>0.05$ ). The main effect of Emotion revealed participants were faster at matching happy than angry faces $(p<0.001)$ and faster at matching fearful than angry faces $(p<0.001)$. There was a non-significant trend for matching fearful faster than happy faces $(p=0.08)$. See Table 2 for behavioral descriptives. 
Table 2 Accuracy and reaction times (in milliseconds) for accurate trials (Mean \pm SD)

\begin{tabular}{|c|c|c|c|c|}
\hline Contrast & gSAD $(n=29)$ & $\mathrm{HC}(\mathrm{n}=27)$ & $t(d f=56)$ & $p$ \\
\hline \multicolumn{5}{|l|}{ Accuracy } \\
\hline Attend Angry vs. Shapes & $85.6 \pm 11.8$ & $86.6 \pm 10.9$ & 0.31 & 0.76 \\
\hline Attend Fear vs. Shapes & $92.7 \pm 9.1$ & $90.7 \pm 10.1$ & 0.75 & 0.46 \\
\hline Attend Happy vs. Shapes & $90.8 \pm 11.0$ & $86.3 \pm 14.2$ & 1.34 & 0.19 \\
\hline Attend Shapes vs. Angry (distractor) & $94.1 \pm 8.1$ & $93.7 \pm 8.0$ & 0.20 & 0.84 \\
\hline Attend Shapes vs. Fear (distractor) & $90.1 \pm 14.4$ & $95.5 \pm 7.6$ & 1.75 & 0.09 \\
\hline Attend Shapes vs. Happy (distractor) & $91.2 \pm 11.5$ & $93.4 \pm 7.5$ & 0.81 & 0.42 \\
\hline \multicolumn{5}{|l|}{ Reaction Times } \\
\hline Attend Angry vs. Shapes & $1479.6 \pm 405.8$ & $1419.9 \pm 219.5$ & 0.68 & 0.50 \\
\hline Attend Fear vs. Shapes & $1337.9 \pm 371.9$ & $1285.7 \pm 218.5$ & 0.63 & 0.53 \\
\hline Attend Happy vs. Shapes & $1303.7 \pm 340.4$ & $1231.0 \pm 207.4$ & 0.96 & 0.34 \\
\hline Attend Shapes vs. Angry (distractor) & $1021.1 \pm 273.5$ & $957.8 \pm 122.4$ & 1.11 & 0.27 \\
\hline Attend Shapes vs. Fear (distractor) & $985.4 \pm 239.9$ & $946.2 \pm 144.4$ & 0.74 & 0.47 \\
\hline Attend Shapes vs. Happy (distractor) & $975.6 \pm 236.4$ & $944.3 \pm 157.7$ & 0.58 & 0.57 \\
\hline
\end{tabular}

\section{Behavioral data: match shapes}

Regarding accuracy, there were no main effects for (distractor) Emotion or Group and no evidence of a (distractor) Emotion x Group interaction (all $p_{s}>0.05$ ). All participants exhibited similar accuracy when matching shapes alongside angry, fearful, and happy distractor faces. Results for RT were analogous as there were no main effects for (distractor) Emotion or Group and no (distractor) Emotion x Group interaction (all $p_{s}>0.05$ ). Participants showed comparable RT among each distractor emotion: shapesangry, shapes-fearful, and shapes-happy (all $p_{s}>0.05$ ). See Table 2 for behavioral descriptives.

\section{Functional MRI}

An ANOVA revealed a main effect of Group for anterior insula and ACC (Table 3). Post hoc t-tests showed greater bilateral anterior insula activity for gSAD versus $\mathrm{HC}$ [left $(-32,22,8), \quad$ F-score $=12.02, \quad$ volume $=560 \mathrm{~mm}^{3}$, svc corrected $p<0.01$; right $(32,26,4)$, F-score $=8.03$, volume $=440 \mathrm{~mm}^{3}$, svc corrected $p<0.03$ ] when attending to emotional faces (vs. shapes) (Figure 2). Conversely, gSAD showed less activation in left rostral ACC (rACC) compared to $\mathrm{HC}[(-6,30,-6), \mathrm{F}$-score $=6.72$, volume $=$ $368 \mathrm{~mm}^{3}$, svc corrected $\left.p<0.05\right]$ when attending to shapes (vs. emotional faces) (Figure 3). The main effect of Emotion and the Group $\mathrm{x}$ Emotion interaction were nonsignificant in a priori regions.

\section{Correlational analyses}

Extent of anterior insula (aINS) reactivity in gSAD corresponded with the intensity of social anxiety symptoms (LSAS-total score) though significance was evident in the left aINS $(\mathrm{r}=0.32, p<0.04$, one-tailed $)$ but not right aINS $(\mathrm{r}=0.20, p=0.1$, one-tailed). No correlations emerged for aINS reactivity and general anxiety (i.e., trait anxiety; [64] or depression level [62] (all $p_{s}>0.05$ ) in the gSAD group.

We observed a negative relationship between both left aINS and rostral ACC $(\mathrm{r}=-0.35, p<0.009$, two-tailed $)$ and right aINS and rostral ACC $(\mathrm{r}=-0.45, p<0.001$, two-tailed) (Figure 4). Of note, these correlations remained significant even after the removal of the $\mathrm{HC}$ and gSAD outliers.

Table 3 Whole-brain voxel-wise analysis of variance

\begin{tabular}{lccccc}
\hline Region & MNI Coordinates & Volume & $\boldsymbol{F}$ statistic \\
\hline Main Effect of Group & $\mathbf{- 3 2}$ & $\mathbf{2 2}$ & $\mathbf{8}$ & $\mathbf{5 6 0}$ & $\mathbf{1 2 . 0 2}$ \\
Anterior insula & $\mathbf{3 2}$ & $\mathbf{2 6}$ & $\mathbf{4}$ & $\mathbf{4 4 0}$ & $\mathbf{8 . 0 3}$ \\
& $\mathbf{- 6}$ & $\mathbf{3 0}$ & $\mathbf{- 6}$ & $\mathbf{3 6 8}$ & $\mathbf{6 . 7 2}$ \\
Anterior cingulate cortex & -30 & -98 & -4 & 104 & 16.03 \\
Middle occipital gyrus & 28 & -86 & $\mathbf{4}$ & 248 & 13.44 \\
Supramarginal gyrus & -64 & -42 & 32 & 224 & 14.15 \\
Supplementary motor area & 8 & -16 & 52 & 128 & 12.43 \\
Main Effect of Emotion & & & & & \\
Hippocampus & -34 & -14 & -12 & 104 & 9.30 \\
Inferior frontal operculum & -36 & 10 & 26 & 112 & 8.73 \\
Group x Emotion Interaction & & & & & \\
Superior frontal gyrus & -18 & 32 & 54 & 1080 & 12.01 \\
Caudate & 16 & -16 & 24 & 184 & 9.91 \\
& 22 & 0 & 24 & 152 & 8.72 \\
Middle frontal gyrus & 40 & -2 & 62 & 104 & 8.48 \\
\hline A proi
\end{tabular}

A priori areas within the atlas-based anatomical regions of interest are shown in bold.

MNI, Montreal Neurological Institute. 

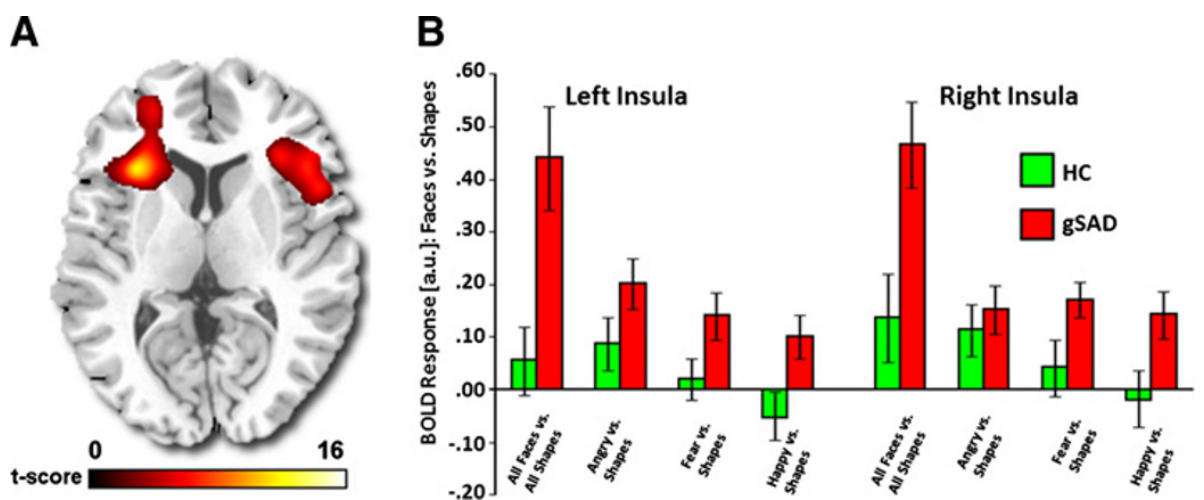

Figure 2 A priori region of interest: Anterior insula. A) Voxel-wise main effect of group for the contrast Match Faces $>$ Match Shapes, along with Match Angry > Match Shapes, Match Fear > Match Shapes, and Match Happy > Match Shapes, showing bilateral anterior insula (alNS) displayed on statistical F-map at $p<0.05$; cluster size $>10$ contiguous voxels (family-wise error corrected for multiple comparisons across small volumes of interest). Color scale reflects F-score. B) Bar graphs depicting extracted parameter estimates of activation from the alNS ROI within each group showing Generalized Social Anxiety Disorder exhibited greater bilateral anterior insula activation than Healthy Controls ( $p<0.05)$.

\section{Discussion}

In this functional MRI study, we examined amygdala, anterior insula (aINS), and anterior cingulate cortex (ACC) activation in patients with generalized social anxiety disorder (gSAD) and demographically matched healthy controls (HC) with a novel "Emotional Faces Shifting Attention Task" (EFSAT). The paradigm is an adaptation of widely used emotional faces matching tasks known to evoke exaggerated amygdala and/or insula reactivity in gSAD $[17,18]$. By spatially combining the traditionally separate faces-only and shapes-only image trials into one trial within one field of view, attention was modulated by having it directed towards or away from emotional faces in order to complete the matching task.
The primary objective in this study was to evaluate attentional modulation with EFSAT in patients with gSAD. When attending to emotional content (Match Faces > Match Shapes), we hypothesized individuals with gSAD relative to $\mathrm{HC}$ would exhibit exaggerated amygdala and aINS reactivity particularly to threat expressions (angry, fearful). Our prediction was partially supported. Though no group effects emerged for amygdala, participants with gSAD compared to HC showed bilateral aINS hyperreactivity when attending to faces, regardless of emotional valence. We also expected a positive correlation between subcortical reactivity and symptom intensity in gSAD. In support, greater left aINS reactivity to emotional faces corresponded with greater symptom severity in gSAD but not with general anxiety or depression
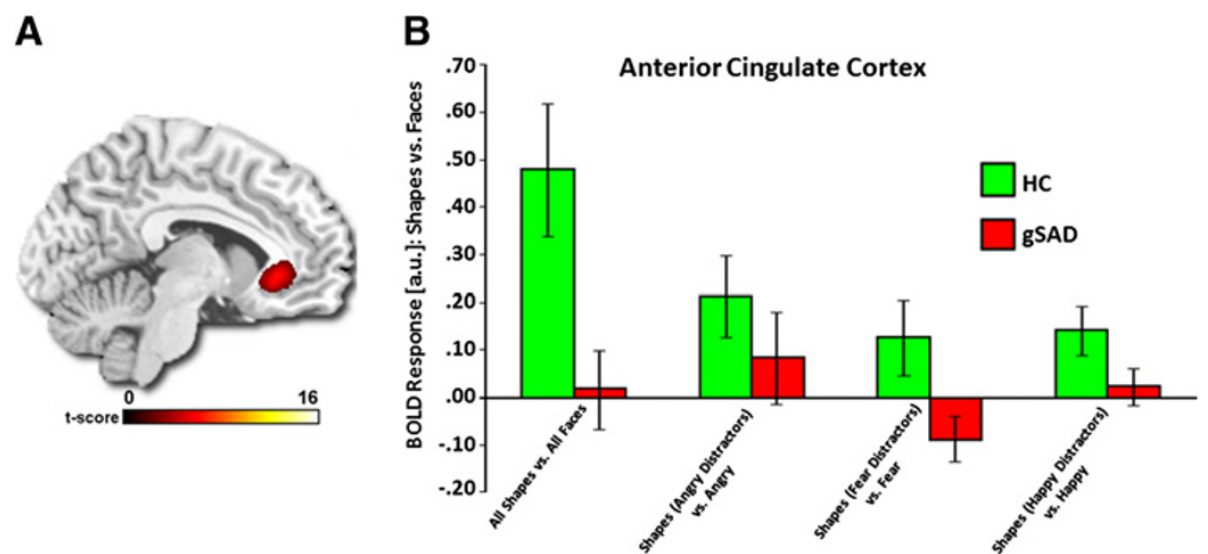

Figure 3 A priori region of interest: Anterior cingulate cortex. A) Voxel-wise main effect of group for the contrast Match Shapes $>$ Match Faces, along with Match Shapes (Angry distractor) > Match Angry, Match Shapes (Fear distractor) > Match Fear, and Match Shapes (Happy distractor) > Match Happy, showing anterior cingulate cortex (ACC) displayed on statistical F-map at $p<0.05$; cluster size $>10$ contiguous voxels (family-wise error corrected for multiple comparisons across small volumes of interest). Color scale reflects F-score. B) Bar graphs depicting extracted parameter estimates of activation from the ACC ROI activation within each group showing Healthy Controls exhibited ACC activation, whereas, Generalized Social Anxiety Disorder showed no 'activation'. 


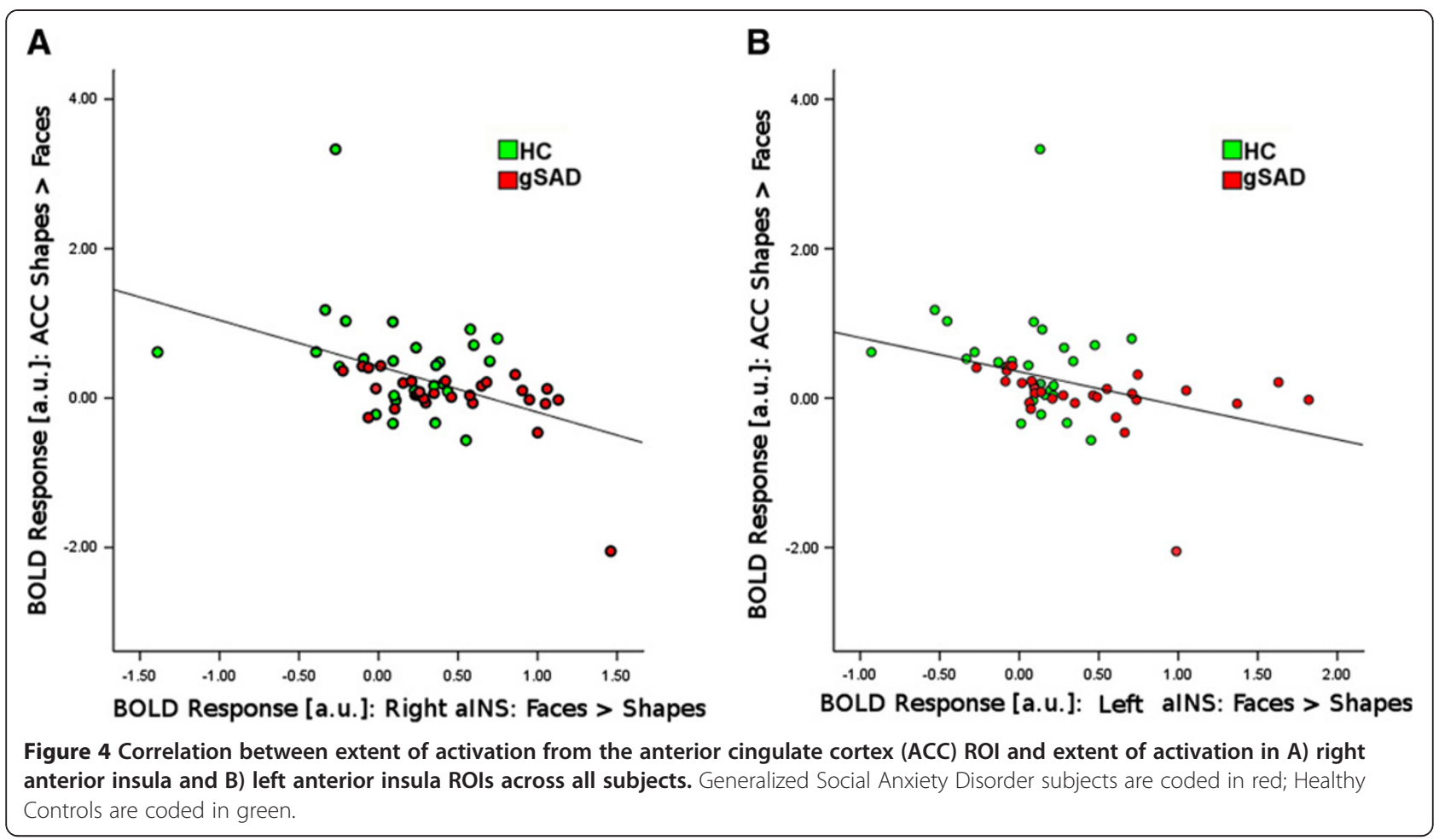

level; however, results were weak (i.e., only revealed with one-tailed t-test). Nevertheless, overall findings substantiate the proposal that interoception, a function of aINS that aids in bringing bodily state to awareness [30,31], underlies anxiety by means of negative beliefs that facilitate, or are the result of, exaggerated interoceptive signals $[28,29]$.

When attending away from emotional faces (Match Shapes $>$ Match Faces), we hypothesized gSAD relative to $\mathrm{HC}$ would exhibit reduced ACC engagement and that deficient ACC response would be most pronounced in the presence of distracting threat faces. Evidence of enhanced rostral ACC in our HC group is consistent with its role in regulatory control $[40,46,65,66]$. Specifically, its recruitment when attention was shifted to shapes indicates it served a gating function in modulating the processing of face distractors. We did not find evidence of group effects for dorsal ACC, another region involved in the inhibition of emotional processing $[45,66]$ suggesting EFSAT may not tap into regulatory regions associated with conflict resolution or stimulus appraisal [66]. Again, our hypothesis was partially supported in that gSAD showed a lack of rostral ACC response; however, there was no interaction with the emotional valence of task-irrelevant faces. To our knowledge, the only other study of implicit attentional control in gSAD comprised an event-related emotional conflict task, an emotional counting Stroop paradigm. Results showed patients with gSAD or generalized anxiety disorder lacked dorsal ACC recruitment to congruent and incongruent negative pictures compared to healthy volunteers [49]. Methodological differences do not premit direct comparison with our study, however, evidence of deficient ACC activation during conflict resolution or sustained goal-directed attention suggests impoverished ACC plays a role in excessive attention to emotional information in gSAD and potentially other disorders of emotion dysregulation.

Across participants, we hypothesized a negative relationship between ACC response during attentional control and subcortical (amygdala and/or aINS) reactivity to emotional faces. The prediction was supported, but only in aINS and not amygdala. In a previous study, we showed greater aINS reactivity to threat faces in gSAD than controls, with reduced functional connectivity between aINS and dorsal ACC activity in gSAD indicating exaggerated aINS reactivity was due to a deficiency in 'top down' control or appraisal [23]. Here, we observe a negative aINS-ACC relationship across subjects based on direct and indirect emotion processing, respectively, along with evidence of enhanced rostral ACC and reduced bilateral aINS activation in the HC group. Given that the ACC is thought to interact with and/ or modulate aINS response to salient signals, we interpret that individuals who exhibit deficient ACC engagement when attentional control is required are those who are likely to exhibit enhanced aINS reactivity when their attention is directed towards emotional stimuli. 


\section{Conclusions}

Findings should be considered in the context of several important limitations. Due to the lack of a baseline (e.g., fixation) condition, findings cannot be interpreted in relation to a change from a non-cognitive and non-emotional task. The lack of 'neutral' target expressions does not permit dissociation between face and emotion-processing influences. Additionally, the paradigm failed to elicit differential amygdala response between gSAD and controls and insula hyper-reactivity in gSAD was not specific to threat. Together, the task may, in these aspects, not be as sensitive as traditional perceptual matching paradigms. Interestingly, subjects with gSAD have been shown to judge happy faces as less approachable than controls [67]. Although we did not measure potential interpretation bias, we cannot rule out the possibility that insula activation in GSAD involved a less positive judgment of happy faces. Lastly, the gSAD group exhibited greater levels of depression and general anxiety (i.e., trait anxiety) than controls; therefore, we cannot rule out potential influences of depression and general anxiety on results.

Despite limitations, these findings suggest a simple, volitional shift in attention to and away from emotional faces differentiates patients with gSAD from healthy controls in terms of engagement of regions integral to emotion processing and attentional control. During direct and indirect processing of emotional faces, gSAD patients exhibit exaggerated insula reactivity and deficient ACC recruitment, respectively, and different valences, both positive and negative, of socio-emotional information.

\section{Competing interests}

The authors declare that they have no competing interests.

\section{Authors' contributions}

HK conceived and designed the study, conducted data analyses and interpretation, and wrote the manuscript. DP and DAF conducted data analyses and interpretation, and participated in drafting the manuscript. MA helped design the study, collected the data, and participated in drafting the manuscript. KLP conceived and designed the study, conducted data interpretation, and participated in drafting the manuscript. All authors read and approved the final manuscript.

\section{Acknowledgements}

This work was supported by grants from the National Institutes of Health, National Institute of Mental Health (MH076198 to KLP and MH093679 to HK).

\begin{abstract}
Author details
'Mood and Anxiety Disorders Research Program, Department of Psychiatry (HK, DP, DAF, KLP), University of Illinois at Chicago, 1747 W. Roosevelt Rd, Chicago, IL 60608, USA. ${ }^{2}$ Department of Psychiatry (MA, KLP), University of Michigan, Ann Arbor, MI, USA. ${ }^{3}$ Neuropsychiatric Research Program (KLP), Mental Health Service, Jesse Brown VA Medical Center, Chicago, IL, USA.
\end{abstract}

Received: 1 December 2012 Accepted: 7 February 2013

Published: 2 April 2013

\section{References}

1. Kessler RC, Chiu WT, Demler O, Merikangas KR, Walters EE: Prevalence, severity, and comorbidity of 12-month DSM-IV disorders in the National Comorbidity Survey Replication. Arch Gen Psychiatry 2005, 62:617-627.

2. Stein MB, Stein DJ: Social anxiety disorder. Lancet 2008, 371:1115-1125.
3. Kessler RC, McGonagle KA, Zhao S, Nelson CB, Hughes M, Eshleman S, Wittchen $\mathrm{H}-\mathrm{U}$, Kendler KS: Lifetime and 12-month prevalence of DSM-III-R psychiatric disorders in the United States: results from the national comorbidity survey. Arch Gen Psychiatry 1994, 51:8-19.

4. Ruscio AM, Brown TA, Chiu WT, Sareen J, Stein MB, Kessler RC: Social fears and social phobia in the USA: results from the National Comorbidity Survey Replication. Psychol Med 2008, 38:15-28.

5. Bögels SM, Mansell W: Attention processes in the maintenance and treatment of social phobia: hypervigilance, avoidance and self-focused attention. Clin Psychol Rev 2004, 24:827-856.

6. Cornwell BR, Johnson L, Berardi L, Grillon C: Anticipation of public speaking in virtual reality reveals a relationship between trait social anxiety and startle reactivity. Biol Psychiatry 2006, 59:664-666.

7. Davidson RJ: Affective style, psychopathology, and resilience: brain mechanisms and plasticity. Am Psychol 2000, 55:1196-1214.

8. McTeague LM, Lang PJ, Laplante M-C, Cuthbert BN, Strauss CC, Bradley MM: Fearful imagery in social phobia: generalization, comorbidity, and physiological reactivity. Biol Psychiatry 2009, 65:374-382.

9. Heimberg RG: Cognitive assessment strategies and the measurement of outcome of treatment for social phobia. Behav Res Ther 1994, 32:269-280.

10. Adolphs R, Tranel D, Damasio H, Damasio AR: Fear and the human amygdala. J Neurosci 1995, 15:5879-5891.

11. Davis $M$, Whalen PJ: The amygdala: vigilance and emotion. Mol Psychiatry 2001, 6:13-34

12. Phan KL, Wager T, Taylor SF, Liberzon I: Functional neuroanatomy of emotion: a meta-analysis of emotion activation studies in PET and fMRI. Neurolmage 2002, 16:331-348.

13. Hariri AR, Whalen PJ: The amygdala: inside and out. F1000 Biol Rep 2011, 3:2.

14. Hariri AR, Tessitore A, Mattay VS, Fera F, Weinberger DR: The amygdala response to emotional stimuli: a comparison of faces and scenes. Neurolmage 2002, 17:317-323.

15. Hariri AR, Drabant EM, Munoz KE, Kolachana BS, Mattay VS, Egan MF, Weinberger DR: A susceptibility gene for affective disorders and the response of the human amygdala. Arch Gen Psychiatry 2005, 62:146-152.

16. Sergerie $K$, Chochol C, Armony JL: The role of the amygdala in emotional processing: a quantitative meta-analysis of functional neuroimaging studies. Neurosci Biobehav Rev 2008, 32:811-830.

17. Etkin A, Wager TD: Functional neuroimaging of anxiety: a meta-analysis of emotional processing in PTSD, Social Anxiety Disorder, and Specific Phobia. Am J Psychiatry 2007, 164:1476-1488.

18. Freitas-Ferrari MC, Hallak JEC, Trzesniak C, Filho AS, Machado-de-Sousa JP, Chagas MHN, Nardi AE, Crippa JAS: Neuroimaging in social anxiety disorder: a systematic review of the literature. Prog Neuropsychopharmacol Biol Psychiatry 2010, 34:565-580.

19. Phan KL, Fitzgerald DA, Nathan PJ, Tancer ME: Association between amygdala hyperactivity to harsh faces and severity of social anxiety in generalized social phobia. Biol Psychiatry 2006, 59:424-429.

20. Amir N, Klumpp H, Elias J, Bedwell JS, Yanasak N, Miller LS: Increased activation of the anterior cingulate cortex during processing of disgust faces in individuals with social phobia. Biol Psychiatry 2005, 57:975-981

21. Ball TM, Sullivan S, Flagan T, Hitchcock CA, Simmons A, Paulus MP, Stein MB: Selective effects of social anxiety, anxiety sensitivity, and negative affectivity on the neural bases of emotional face processing. Neurolmage 2012, 59:1879-1887.

22. Evans KC, Wright Cl, Wedig MM, Gold AL, Pollack MH, Rauch SL: A functional MRI study of amygdala responses to angry schematic faces in social anxiety disorder. Depress Anxiety 2008, 25:496-505.

23. Klumpp $\mathrm{H}$, Angstadt M, Phan KL: Insula reactivity and connectivity to anterior cingulate cortex when processing threat in generalized social anxiety disorder. Biol Psychol 2012, 89:273-276.

24. Schmidt S, Mohr A, Miltner WHR, Straube T: Task-dependent neural correlates of the processing of verbal threat-related stimuli in social phobia. Biol Psychol 2010, 84:304-312

25. Shah SG, Klumpp H, Angstadt M, Nathan PJ, Phan KL: Amygdala and insula response to emotional images in patients with generalized social anxiety disorder. J Psychiatry Neurosci 2009, 34:296-302.

26. Straube T, Kolassa I-T, Glauer M, Mentzel H-J, Miltner WHR: Effect of task conditions on brain responses to threatening faces in social phobics: an event-related functional magnetic resonance imaging study. Biol Psychiatry 2004, 56:921-930. 
27. Straube T, Mentzel H-J, Miltner WHR: Common and distinct brain activation to threat and safety signals in social phobia. Neuropsychobiology 2005, 52:163-168.

28. Paulus MP, Stein MB: An insular view of anxiety. Biol Psychiatry 2006, 60:383-387

29. Paulus MP, Stein MB: Interoception in anxiety and depression. Brain Struct Funct 2010, 214:451-463.

30. Craig ADB: How do you feel-now? The anterior insula and human awareness. Nat Rev Neurosci 2009, 10:59-70.

31. Jones CL, Ward J, Critchley HD: The neuropsychological impact of insular cortex lesions. J Neurol Neurosurg Psychiatr 2010, 81:611-618.

32. Amaral DG, Price JL: Amygdalo-cortical projections in the monkey (Macaca fascicularis). J Comp Neurol 1984, 230:465-496.

33. Ghashghaei HT, Barbas H: Pathways for emotion: interactions of prefrontal and anterior temporal pathways in the amygdala of the rhesus monkey. Neuroscience 2002, 115:1261-1279.

34. Ghashghaei HT, Hilgetag CC, Barbas H: Sequence of information processing for emotions based on the anatomic dialogue between prefrontal cortex and amygdala. Neurolmage 2007, 34:905-923.

35. Augustine JR: Circuitry and functional aspects of the insular lobe in primates including humans. Brain Res Brain Res Rev 1996, 22:229-244.

36. Craig AD: How do you feel? interoception: the sense of the physiological condition of the body. Nat Rev Neurosci 2002, 3:655-666.

37. Mesulam MM, Mufson EJ: Insula of the old world monkey. III: efferent cortical output and comments on function. J Comp Neurol 1982, 212:38-52.

38. Nelson SM, Dosenbach NUF, Cohen AL, Wheeler ME, Schlaggar BL, Petersen SE: Role of the anterior insula in task-level control and focal attention. Brain Struct Funct 2010, 214:669-680.

39. Seeley WW, Menon V, Schatzberg AF, Keller J, Glover GH, Kenna H, Reiss AL, Greicius MD: Dissociable intrinsic connectivity networks for salience processing and executive control. J Neurosci 2007, 27:2349-2356.

40. Bishop S, Duncan J, Brett M, Lawrence AD: Prefrontal cortical function and anxiety: controlling attention to threat-related stimuli. Nat Neurosci 2004, 7:184-188.

41. Bishop SJ, Jenkins R, Lawrence AD: Neural processing of fearful faces: effects of anxiety are gated by perceptual capacity limitations. Cereb Cortex 2007, 17:1595-1603.

42. Botvinick MM, Braver TS, Barch DM, Carter CS, Cohen JD: Conflict monitoring and cognitive control. Psychol Rev 2001, 108:624-652.

43. Carter CS, Braver TS, Barch DM, Botvinick MM, Noll D, Cohen JD: Anterior cingulate cortex, error detection, and the online monitoring of performance. Science 1998, 280:747-749.

44. Duncan J, Owen AM: Common regions of the human frontal lobe recruited by diverse cognitive demands. Trends Neurosci 2000, 23:475-483.

45. Etkin A, Egner T, Peraza DM, Kandel ER, Hirsch J: Resolving emotional conflict: a role for the rostral anterior cingulate cortex in modulating activity in the amygdala. Neuron 2006, 51:871-882.

46. Kanske P, Kotz SA: Emotion speeds up conflict resolution: a new role for the ventral anterior cingulate cortex? Cereb Cortex 2011, 21:911-919.

47. MacDonald AW III, Cohen JD, Stenger VA, Carter CS: Dissociating the role of the dorsolateral prefrontal and anterior cingulate cortex in cognitive control. Science 2000, 288:1835-1838.

48. Whalen PJ, Bush G, Shin LM, Rauch SL: The emotional counting Stroop: a task for assessing emotional interference during brain imaging. Nat Protoc 2006, 1:293-296.

49. Blair KS, Geraci M, Smith BW, Hollon N, Devido J, Otero M, Blair JR, Pine DS: Reduced dorsal anterior cingulate cortical activity during emotional regulation and top-down attentional control in generalized social phobia, generalized anxiety disorder, and comorbid generalized social phobia/generalized anxiety disorder. Biol Psychiatry 2012, 72:476-482

50. Shin LM, Whalen PJ, Pitman RK, Bush G, Macklin ML, Lasko NB, Orr SP, Mclnerney SC, Rauch SL: An fMRI study of anterior cingulate function in posttraumatic stress disorder. Biol Psychiatry 2001, 50:932-942.

51. Carlson JM, Reinke KS, Habib R: A left amygdala mediated network for rapid orienting to masked fearful faces. Neuropsychologia 2009, 47:1386-1389.

52. Pourtois G, Schwartz S, Seghier ML, Lazeyras F, Vuilleumier P: Neural systems for orienting attention to the location of threat signals: an event-related fMRI study. Neurolmage 2006, 31:920-933.

53. Vuilleumier P, Armony JL, Driver J, Dolan RJ: Effects of attention and emotion on face processing in the human brain: an event-related fMRI study. Neuron 2001, 30:829-841.
54. Pessoa L, Kastner S, Ungerleider LG: Attentional control of the processing of neutral and emotional stimuli. Brain Res Cogn Brain Res 2002, 15:31-45.

55. Klumpp H, Ho SS, Taylor SF, Phan KL, Abelson JL, Liberzon I: Trait anxiety modulates anterior cingulate activation to threat interference. Depress Anxiety 2011, 28:194-201.

56. Krug MK, Carter CS: Adding fear to conflict: a general purpose cognitive control network is modulated by trait anxiety. Cogn Affect Behav Neurosci 2010, 10:357-371.

57. Price RB, Eldreth DA, Mohlman J: Deficient prefrontal attentional control in late-life generalized anxiety disorder: an fMRI investigation. Trans/ Psychiatry 2011, 1:e46.

58. Hajcak G, Olvet DM: The persistence of attention to emotion: brain potentials during and after picture presentation. Emotion 2008, 8:250-255

59. Klumpp $\mathrm{H}$, Angstadt $\mathrm{M}$, Phan $\mathrm{KL}$ : Shifting the focus of attention modulates amygdala and anterior cingulate cortex reactivity to emotional faces. Neurosci Lett 2012, 514:210-213.

60. First MB, Spitzer RL, Gibbon M, Williams JBW: Structured Clinical Interview for the DSM-IV Axis I Disorders: SCID-I/P, Version 2.0.: Biometrics Research Department, New York State Psychiatric Institute; 1997.

61. Liebowitz MR: Social phobia. Mod Probl Pharmacopsychiatry 1987, 22:141-173.

62. Beck AT, Steer RA, Brown GK: Manual for the Beck Depression Inventory-II. San Antonio, TX: Psychological Corp; 1996.

63. Gur RC, Schroeder L, Turner T, McGrath C, Chan RM, Turetsky BI, Alsop D, Maldjian J, Gur RE: Brain activation during facial emotion processing. Neurolmage 2002, 16:651-662.

64. Spielberger CD, Gorsuch RL, Lushene R, Vagg PR, Jacobs GA: Manual for the State-Trait Anxiety Inventory. Palo Alto, CA: Consulting Psychologists Press; 1983.

65. Bush G, Luu P, Posner Ml: Cognitive and emotional influences in anterior cingulate cortex. Trends Cogn Sci 2000, 4:215-222.

66. Etkin A, Egner $\mathrm{T}$, Kalisch R: Emotional processing in anterior cingulate and medial prefrontal cortex. Trends Cogn Sci 2011, 15:85-93.

67. Campbell DW, Sareen J, Stein MB, Kravetsky LB, Paulus MP, Hassard ST, Reiss JP: Happy but not so approachable: the social judgments of individuals with generalized social phobia. Depress Anxiety 2009, 26:419-424.

doi:10.1186/2045-5380-3-7

Cite this article as: Klumpp et al: Anterior cingulate cortex and insula response during indirect and direct processing of emotional faces in generalized social anxiety disorder. Biology of Mood \& Anxiety Disorders 2013 3:7.

\section{Submit your next manuscript to BioMed Central and take full advantage of:}

- Convenient online submission

- Thorough peer review

- No space constraints or color figure charges

- Immediate publication on acceptance

- Inclusion in PubMed, CAS, Scopus and Google Scholar

- Research which is freely available for redistribution

Submit your manuscript at www.biomedcentral.com/submit
C) Biomed Central 